\title{
Generalizations of some fractional integral inequalities for $m$-convex functions via generalized Mittag-Leffler function
}

\author{
Ghulam Farid and Ghulam Abbas
}

\begin{abstract}
In this paper we are interested to present some general fractional integral inequalities for $m$-convex functions by involving generalized Mittag-Leffler function. In particular we produce inequalities for several kinds of fractional integrals. Also these inequalities have some connections with known integral inequalities.
\end{abstract}

Mathematics Subject Classification (2010): 26A51, 26A33, 33E12.

Keywords: $m$-convex function, Hadamard inequality, generalized Mittag-Leffler function.

\section{Introduction}

Inequalities play an essential role in mathematical and other kinds of analysis, specially inequalities involving derivative and integral of functions are of great interest for researchers.

Convex functions are very special in the study of functions defined on real line, a lot of results, in particular inequalities in mathematical analysis based on their invention. A convex function $f: I \rightarrow \mathbb{R}$ is also equivalently defined by the Hadamard inequality

$$
f\left(\frac{a+b}{2}\right) \leq \frac{1}{b-a} \int_{a}^{b} f(t) d t \leq \frac{f(a)+f(b)}{2}
$$

where $a, b \in I, a<b$.

A close generalized form of convex functions is $m$-convex functions introduced by Toader [23].

Definition 1.1. A function $f:[0, b] \rightarrow \mathbb{R}, b>0$ is said to be $m$-convex function if for all $x, y \in[0, b]$ and $t \in[0,1]$

$$
f(t x+m(1-t) y) \leq t f(x)+m(1-t) f(y)
$$


holds for $m \in[0,1]$.

Every $m$-convex function is not convex function.

Example 1.2. [16] Let $f:[0, \infty] \rightarrow \mathbb{R}$ be defined by

$$
g(t)=\frac{1}{12}\left(x^{4}-5 x^{3}+9 x^{2}-5 x\right)
$$

is $\frac{16}{17}$-convex function but it is not convex function.

For $m=1$ the above definition becomes the definition of convex functions defined on $[0, b]$. If we take $m=0$, then we obtain the concept of starshaped functions on $[0, b]$. A function $f:[0, b] \rightarrow \mathbb{R}$ is said to be starshaped if $f(t x) \leq t f(x)$ for all $t \in[0,1]$ and $x \in[0, b]$.

If set of $m$-convex functions on $[0, b]$ for which $f(0)<0$ is denoted by $K_{m}(b)$, then we have

$$
K_{1}(b) \subset K_{m}(b) \subset K_{0}(b)
$$

whenever $m \in(0,1)$. In the class $K_{1}(b)$ there are convex functions $f:[0, b] \rightarrow \mathbb{R}$ for which $f(0) \leq 0$ (see, $[2]$ ). There are a number of results and inequalities obtained via $m$-convex functions for detail (see $[2,4,7,10]$ ).

Recently, a number of authors are taking keen interest to obtain integral inequalities of the Hadamard type via fractional integral operators of different kinds in the various field of fractional calculus. For example one can see $[5,6,11,15,17,20,22]$.

\section{Preliminaries in fractional calculus and integral operators}

Fractional calculus deals with the study of integral and differential operators of non-integral order. Many mathematicians like Liouville, Riemann and Weyl made major contributions to the theory of fractional calculus. The study on the fractional calculus continued with contributions from Fourier, Abel, Lacroix, Leibniz, Grunwald and Letnikov. For detail (see, $[11,13,15])$. Riemann-Liouville fractional integral operator is the first formulation of an integral operator of non-integral order.

Definition 2.1. [24] Let $f \in L_{1}[a, b]$. Then Riemann-Liouville fractional integral of $f$ of order $\nu$ is defined by

$$
I_{a^{+}}^{\nu} f(x)=\frac{1}{\Gamma(\nu)} \int_{a}^{x}(x-t)^{\nu-1} f(t) d t, x>a
$$

and

$$
I_{b_{-}}^{\nu} f(x)=\frac{1}{\Gamma(\nu)} \int_{x}^{b}(t-x)^{\nu-1} f(t) d t, x<b .
$$

In fact these formulations of fractional integral operators have been established due to Letnikov [14], Sonin [21] and then by Laurent [12]. In these days a variety of fractional integral operators have been produced and many are under discussion. A number of generalized fractional integral operators are also very useful in generalizing the theory of fractional integral operators $[1,11,15,18,22,24]$. 
Definition 2.2. [18] Let $\mu, \nu, k, l, \gamma$ be positive real numbers and $\omega \in \mathbb{R}$. Then the generalized fractional integral operators containing Mittag-Leffler function $\epsilon_{\mu, \nu, l, \omega, a^{+}}^{\gamma, \delta, k}$ and $\epsilon_{\mu, \nu, l, \omega, b_{-}}^{\gamma, \delta, k}$ for a real valued continuous function $f$ is defined by:

$$
\left(\epsilon_{\mu, \nu, l, \omega, a^{+}}^{\gamma, \delta, k} f\right)(x)=\int_{a}^{x}(x-t)^{\nu-1} E_{\mu, \nu, l}^{\gamma, \delta, k}\left(\omega(x-t)^{\mu}\right) f(t) d t
$$

and

$$
\left(\epsilon_{\mu, \nu, l, \omega, b_{-}}^{\gamma, \delta, k} f\right)(x)=\int_{x}^{b}(t-x)^{\nu-1} E_{\mu, \nu, l}^{\gamma, \delta, k}\left(\omega(t-x)^{\mu}\right) f(t) d t,
$$

where the function $E_{\mu, \nu, l}^{\gamma, \delta, k}$ is generalized Mittag-Leffler function defined as

$$
E_{\mu, \nu, l}^{\gamma, \delta, k}(t)=\sum_{n=0}^{\infty} \frac{(\gamma)_{k n} t^{n}}{\Gamma(\mu n+\nu)(\delta)_{l n}}
$$

$(a)_{n}$ is the Pochhammer symbol, it defined as

$$
(a)_{n}=a(a+1)(a+2) \ldots(a+n-1),(a)_{0}=1 .
$$

If $\delta=l=1$ in (2.1), then integral operator $\epsilon_{\mu, \nu, l, \omega, a^{+}}^{\gamma, \delta, k}$ reduces to an integral operator $\epsilon_{\mu, \nu, 1, \omega, a^{+}}^{\gamma, 1, k}$ containing generalized Mittag-Leffler function $E_{\mu, \nu, 1}^{\gamma, 1, k}$ introduced by Srivastava and Tomovski in [22]. Along with $\delta=l=1$ in addition if $k=1$ then (2.1) reduces to an integral operator defined by Prabhaker in [17] containing Mittag-Leffler function $E_{\mu, \nu}^{\gamma}$. For $\omega=0$ in (2.1), integral operator $\epsilon_{\mu, \nu, l, \omega, a^{+}}^{\gamma, \delta, k}$ reduces to the Riemann-Liouville fractional integral operator [18].

In $[18,22]$ properties of generalized integral operator and generalized MittagLeffler functions are studied in details. In [18] it is proved that $E_{\mu, \nu, l}^{\gamma, \delta, k}(t)$ is absolutely convergent for $k<l+\mu$. Let $S$ be the sum of series of absolute terms of $E_{\mu, \nu, l}^{\gamma, \delta, k}(t)$. We will use this property of Mittag-Leffler function in sequal.

Now a days a number of authors are working on inequalities involving fractional integral operators and generalized fractional integral operators for example RiemannLiouville, Caputo, Hilfer, Canvati etc [8, 20]. Actually, fractional integral inequalities are very useful to find the uniqueness of solutions for partial differential equations of non-integral order. In this paper we give some fractional integral inequalities for $m$-convex functions by involving generalized Mittag-Leffler function. Also we deduce some main results of $[3,9,19]$.

\section{Fractional integral inequalities} results.

First we prove the following lemma which would be helpful to obtain the main

Lemma 3.1. Let $f: I \rightarrow \mathbb{R}$ be a differentiable mapping on $I, a, b \in I$ with $0 \leq a<b$ and also let $g:[a, m b] \rightarrow \mathbb{R}$ be a continuous function on $[a, m b]$. If $f^{\prime}, g \in L[a, m b]$, 
then the following equality holds for $\nu>0$

$$
\begin{aligned}
& \left(\int_{a}^{m b} g(s) E_{\mu, \nu, l}^{\gamma, \delta, k}\left(\omega s^{\mu}\right) d s\right)^{\nu}[f(a)+f(m b)] \\
& -\nu \int_{a}^{m b}\left(\int_{a}^{t} g(s) E_{\mu, \nu, l}^{\gamma, \delta, k}\left(\omega s^{\mu}\right) d s\right)^{\nu-1} g(t) E_{\mu, \nu, l}^{\gamma, \delta, k}\left(\omega t^{\mu}\right) f(t) d t \\
& -\nu \int_{a}^{m b}\left(\int_{t}^{m b} g(s) E_{\mu, \nu, l}^{\gamma, \delta, k}\left(\omega s^{\mu}\right) d s\right)^{\nu-1} g(t) E_{\mu, \nu, l}^{\gamma, \delta, k}\left(\omega t^{\mu}\right) f(t) d t \\
& =\int_{a}^{m b}\left(\int_{a}^{t} g(s) E_{\mu, \nu, l}^{\gamma, \delta, k}\left(\omega s^{\mu}\right) d s\right)^{\nu} f^{\prime}(t) d t \\
& -\int_{a}^{m b}\left(\int_{t}^{m b} g(s) E_{\mu, \nu, l}^{\gamma, \delta, k}\left(\omega s^{\alpha}\right) d s\right)^{\nu} f^{\prime}(t) d t
\end{aligned}
$$

where $E_{\mu, \nu, l}^{\gamma, \delta, k}$ is generalized Mittag-Leffler function.

Proof. One can have on integrating by parts

$$
\begin{aligned}
& \int_{a}^{m b}\left(\int_{a}^{t} g(s) E_{\mu, \nu, l}^{\gamma, \delta, k}\left(\omega s^{\mu}\right) d s\right)^{\nu} f^{\prime}(t) d t \\
& =\left(\int_{a}^{m b} g(s) E_{\mu, \nu, l}^{\gamma, \delta, k}\left(\omega s^{\mu}\right) d s\right)^{\nu} f(m b) \\
& -\nu \int_{a}^{m b}\left(\int_{a}^{t} g(s) E_{\mu, \nu, l}^{\gamma, \delta, k}\left(\omega s^{\mu}\right) d s\right)^{\nu-1} g(t) E_{\mu, \nu, l}^{\gamma, \delta, k}\left(\omega t^{\mu}\right) f(t) d t .
\end{aligned}
$$

And likewise

$$
\begin{aligned}
& \int_{a}^{m b}\left(\int_{t}^{m b} g(s) E_{\mu, \nu, l}^{\gamma, \delta, k}\left(\omega s^{\mu}\right) d s\right)^{\nu} f^{\prime}(t) d t \\
& =-\left(\int_{a}^{m b} g(s) E_{\mu, \nu, l}^{\gamma, \delta, k}\left(\omega s^{\mu}\right) d s\right)^{\nu} f(a) \\
& +\nu \int_{a}^{m b}\left(\int_{t}^{m b} g(s) E_{\mu, \nu, l}^{\gamma, \delta, k}\left(\omega s^{\mu}\right) d s\right)^{\nu-1} g(t) E_{\mu, \nu, l}^{\gamma, \delta, k}\left(\omega t^{\mu}\right) f(t) d t .
\end{aligned}
$$

On substracting equation (3.3) from (3.2), we get the result.

We use Lemma 3.1 to establish the following fractional integral inequality.

Theorem 3.2. Let $f: I \rightarrow \mathbb{R}$ be a differentiable mapping on $I, a, b \in I$ with $0 \leq a<b$ and also let $g:[a, m b] \rightarrow \mathbb{R}$ be a continuous function on $[a, m b]$. If $\left|f^{\prime}\right|$ is $m$-convex 
function on $[a, m b]$, then the following inequality holds

$$
\begin{aligned}
& \mid\left(\int_{a}^{m b} g(s) E_{\mu, \nu, l}^{\gamma, \delta, k}\left(\omega s^{\mu}\right) d s\right)^{\nu}(f(a)+f(m b)) \\
& -\nu \int_{a}^{m b}\left(\int_{a}^{t} g(s) E_{\mu, \nu, l}^{\gamma, \delta, k}\left(\omega s^{\mu}\right) d s\right)^{\nu-1} g(t) E_{\mu, \nu, l}^{\gamma, \delta, k}\left(\omega t^{\mu}\right) f(t) d t \\
& -\nu \int_{a}^{m b}\left(\int_{t}^{m b} g(s) E_{\mu, \nu, l}^{\gamma, \delta, k}\left(\omega s^{\mu}\right) d s\right)^{\nu-1} g(t) E_{\mu, \nu, l}^{\gamma, \delta, k}\left(\omega t^{\mu}\right) f(t) d t \mid \\
& \leq \frac{(m b-a)^{\nu+1}\|g\|_{\infty}^{\nu} S^{\nu}}{\nu+1}\left(\left|f^{\prime}(a)\right|+m\left|f^{\prime}(b)\right|\right)
\end{aligned}
$$

for $k<l+\mu$, where $\|g\|_{\infty}=\sup _{t \in[a, b]}|g(t)|$.

Proof. By using Lemma 3.1, we have

$$
\begin{aligned}
& \mid\left(\int_{a}^{m b} g(s) E_{\mu, \nu, l}^{\gamma, \delta, k}\left(\omega s^{\mu}\right) d s\right)^{\nu}(f(a)+f(m b)) \\
& -\nu \int_{a}^{m b}\left(\int_{a}^{t} g(s) E_{\mu, \nu, l}^{\gamma, \delta, k}\left(\omega s^{\mu}\right) d s\right)^{\nu-1} g(t) E_{\mu, \nu, l}^{\gamma, \delta, k}\left(\omega t^{\mu}\right) f(t) d t \\
& -\nu \int_{a}^{m b}\left(\int_{t}^{m b} g(s) E_{\mu, \nu, l}^{\gamma, \delta, k}\left(\omega s^{\mu}\right) d s\right)^{\nu-1} g(t) E_{\mu, \nu, l}^{\gamma, \delta, k}\left(\omega t^{\mu}\right) f(t) d t \mid \\
& \leq \int_{a}^{m b}\left|\int_{a}^{t} g(s) E_{\mu, \nu, l}^{\gamma, \delta, k}\left(\omega s^{\mu}\right) d s\right|^{\nu}\left|f^{\prime}(t)\right| d t \\
& +\int_{a}^{m b}\left|\int_{t}^{m b} g(s) E_{\mu, \nu, l}^{\gamma, \delta, k}\left(\omega s^{\mu}\right) d s\right|^{\nu}\left|f^{\prime}(t)\right| d t .
\end{aligned}
$$

By using $\|g\|_{\infty}=\sup _{t \in[a, b]}|g(t)|$ and absolute convergence of Mittag-Leffler function, we have

$$
\begin{aligned}
& \mid\left(\int_{a}^{m b} g(s) E_{\mu, \nu, l}^{\gamma, \delta, k}\left(\omega s^{\mu}\right) d s\right)^{\nu}(f(a)+f(m b)) \\
& -\nu \int_{a}^{m b}\left(\int_{a}^{t} g(s) E_{\mu, \nu, l}^{\gamma, \delta, k}\left(\omega s^{\mu}\right) d s\right)^{\nu-1} g(t) E_{\mu, \nu, l}^{\gamma, \delta, k}\left(\omega t^{\mu}\right) f(t) d t \\
& -\nu \int_{a}^{m b}\left(\int_{t}^{m b} g(s) E_{\mu, \nu, l}^{\gamma, \delta, k}\left(\omega s^{\mu}\right) d s\right)^{\nu-1} g(t) E_{\mu, \nu, l}^{\gamma, \delta, k}\left(\omega t^{\mu}\right) f(t) d t \mid \\
& \leq\|g\|_{\infty}^{\nu} S^{\nu}\left(\int_{a}^{m b}(t-a)^{\nu}\left|f^{\prime}(t)\right| d t+\int_{a}^{m b}(m b-t)^{\nu}\left|f^{\prime}(t)\right| d t\right) .
\end{aligned}
$$


Since $\left|f^{\prime}\right|$ is $m$-convex function, therefore it can be written as

$$
\left|f^{\prime}(t)\right| \leq \frac{m b-t}{m b-a}\left|f^{\prime}(a)\right|+\frac{m(t-a)}{m b-a}\left|f^{\prime}(b)\right|
$$

for $t \in[a, m b]$.

Using (3.6) in (3.5), we have

$$
\begin{aligned}
& \mid\left(\int_{a}^{m b} g(s) E_{\mu, \nu, l}^{\gamma, \delta, k}\left(\omega s^{\mu}\right) d s\right)^{\nu}(f(a)+f(m b)) \\
& -\nu \int_{a}^{m b}\left(\int_{a}^{t} g(s) E_{\mu, \nu, l}^{\gamma, \delta, k}\left(\omega s^{\mu}\right) d s\right)^{\nu-1} g(t) E_{\mu, \nu, l}^{\gamma, \delta, k}\left(\omega t^{\mu}\right) f(t) d t \\
& -\nu \int_{a}^{m b}\left(\int_{t}^{m b} g(s) E_{\mu, \nu, l}^{\gamma, \delta, k}\left(\omega s^{\mu}\right) d s\right)^{\mu-1} g(t) E_{\mu, \nu, l}^{\gamma, \delta, k}\left(\omega t^{\mu}\right) f(t) d t \mid \\
& \leq\|g\|_{\infty}^{\nu} S^{\nu}\left(\int_{a}^{m b}(t-a)^{\nu}\left(\frac{m b-t}{m b-a}\left|f^{\prime}(a)\right|+\frac{m(t-a)}{m b-a}\left|f^{\prime}(b)\right|\right) d t\right. \\
& \left.+\int_{a}^{m b}(m b-t)^{\nu}\left(\frac{m b-t}{m b-a}\left|f^{\prime}(a)\right|+\frac{m(t-a)}{m b-a}\left|f^{\prime}(b)\right|\right) d t\right) .
\end{aligned}
$$

After simplification of above inequality we get the result.

Remark 3.3. By taking particular values of parameters used in Mittag-Leffler function in above theorem several fractional integral inequalities can be obtained for corresponding fractional integrals. For example see the following results.

Corollary 3.4. If we take $m=1$ in Theorem 3.2, then we get the following inequality

$$
\begin{aligned}
& \mid\left(\int_{a}^{b} g(s) E_{\mu, \nu, l}^{\gamma, \delta, k}\left(\omega s^{\mu}\right) d s\right)^{\nu}(f(a)+f(b)) \\
& -\nu \int_{a}^{b}\left(\int_{a}^{t} g(s) E_{\mu, \nu, l}^{\gamma, \delta, k}\left(\omega s^{\mu}\right) d s\right)^{\nu-1} g(t) E_{\mu, \nu, l}^{\gamma, \delta, k}\left(\omega t^{\mu}\right) f(t) d t \\
& -\nu \int_{a}^{b}\left(\int_{t}^{b} g(s) E_{\mu, \nu, l}^{\gamma, \delta, k}\left(\omega s^{\mu}\right) d s\right)^{\nu-1} g(t) E_{\mu, \nu, l}^{\gamma, \delta, k}\left(\omega t^{\mu}\right) f(t) d t \mid \\
& \leq \frac{(b-a)^{\nu+1}\|g\|_{\infty}^{\nu} S^{\nu}}{\nu+1}\left(\left|f^{\prime}(a)\right|+\left|f^{\prime}(b)\right|\right) .
\end{aligned}
$$

Remark 3.5. In Theorem 3.2, for $m=1$.

(i) If we put $\omega=0$, then we get $[19$, Theorem 6$]$.

(ii) If we take $\omega=0, \nu=\frac{\mu}{k}$ and $g(s)=1$, then we get [9, Corollary 2.3].

(iii) For $g(s)=1$ along with $\omega=0$ and $\nu=\mu$, then we get [19, Corollary 2].

Next we give another fractional integral inequality. 
Theorem 3.6. Let $f: I \rightarrow \mathbb{R}$ be a differentiable mapping on $I, a, b \in I$ with $0 \leq a<b$ and also let $g:[a, m b] \rightarrow \mathbb{R}$ be a continuous function on $[a, m b]$. If $\left|f^{\prime}\right|^{q}$ is $m$-convex function on $[a, m b]$ for $q>1$, then the following inequality holds

$$
\begin{aligned}
& \mid\left(\int_{a}^{m b} g(s) E_{\mu, \nu, l}^{\gamma, \delta, k}\left(\omega s^{\mu}\right) d s\right)^{\nu}(f(a)+f(m b)) \\
& -\nu \int_{a}^{m b}\left(\int_{a}^{t} g(s) E_{\mu, \nu, l}^{\gamma, \delta, k}\left(\omega s^{\mu}\right) d s\right)^{\nu-1} g(t) E_{\mu, \nu, l}^{\gamma, \delta, k}\left(\omega t^{\mu}\right) f(t) d t \\
& -\nu \int_{a}^{m b}\left(\int_{t}^{m b} g(s) E_{\mu, \nu, l}^{\gamma, \delta, k}\left(\omega s^{\mu}\right) d s\right)^{\nu-1} g(t) E_{\mu, \nu, l}^{\gamma, \delta, k}\left(\omega t^{\mu}\right) f(t) d t \mid \\
& \leq \frac{2(m b-a)^{\nu+1}\|g\|_{\infty}^{\nu} S^{\nu}}{(\nu p+1)^{\frac{1}{q}}}\left(\frac{\left|f^{\prime}(a)\right|^{q}+m\left|f^{\prime}(b)\right|^{q}}{2}\right)^{\frac{1}{q}}
\end{aligned}
$$

for $k<l+\mu$, where $\|g\|_{\infty}=\sup _{t \in[a, b]}|g(t)|$ and $\frac{1}{p}+\frac{1}{q}=1$.

Proof. By using Lemma 3.1, we have

$$
\begin{aligned}
& \mid\left(\int_{a}^{m b} g(s) E_{\mu, \nu, l}^{\gamma, \delta, k}\left(\omega s^{\mu}\right) d s\right)^{\nu}(f(a)+f(m b)) \\
& -\nu \int_{a}^{m b}\left(\int_{a}^{t} g(s) E_{\mu, \nu, l}^{\gamma, \delta, k}\left(\omega s^{\mu}\right) d s\right)^{\nu-1} g(t) E_{\mu, \nu, l}^{\gamma, \delta, k}\left(\omega t^{\mu}\right) f(t) d t \\
& -\nu \int_{a}^{m b}\left(\int_{t}^{m b} g(s) E_{\mu, \nu, l}^{\gamma, \delta, k}\left(\omega s^{\mu}\right) d s\right)^{\nu-1} g(t) E_{\mu, \nu, l}^{\gamma, \delta, k}\left(\omega t^{\mu}\right) f(t) d t \mid \\
& \leq \int_{a}^{m b}\left|\int_{a}^{t} g(s) E_{\mu, \nu, l}^{\gamma, \delta, k}\left(\omega s^{\mu}\right) d s\right|^{\nu}\left|f^{\prime}(t)\right| d t \\
& +\int_{a}^{m b}\left|\int_{t}^{m b} g(s) E_{\mu, \nu, l}^{\gamma, \delta, k}\left(\omega s^{\mu}\right) d s\right|^{\nu}\left|f^{\prime}(t)\right| d t .
\end{aligned}
$$

Using Hölder inequality, we have

$$
\begin{aligned}
& \mid\left(\int_{a}^{m b} g(s) E_{\mu, \nu, l}^{\gamma, \delta, k}\left(\omega s^{\mu}\right) d s\right)^{\nu}(f(a)+f(m b)) \\
& -\nu \int_{a}^{m b}\left(\int_{a}^{t} g(s) E_{\mu, \nu, l}^{\gamma, \delta, k}\left(\omega s^{\mu}\right) d s\right)^{\nu-1} g(t) E_{\mu, \nu, l}^{\gamma, \delta, k}\left(\omega t^{\mu}\right) f(t) d t \\
& -\nu \int_{a}^{m b}\left(\int_{t}^{m b} g(s) E_{\mu, \nu, l}^{\gamma, \delta, k}\left(\omega s^{\mu}\right) d s\right)^{\nu-1} g(t) E_{\mu, \nu, l}^{\gamma, \delta, k}\left(\omega t^{\mu}\right) f(t) d t \mid
\end{aligned}
$$




$$
\begin{aligned}
& \leq\left(\int_{a}^{m b}\left|\int_{a}^{t} g(s) E_{\mu, \nu, l}^{\gamma, \delta, k}\left(\omega s^{\mu}\right) d s\right|^{\nu p} d t\right)^{\frac{1}{p}}\left(\int_{a}^{m b}\left|f^{\prime}(t)\right|^{q} d t\right)^{\frac{1}{q}} \\
& +\left(\int_{a}^{m b}\left|\int_{t}^{m b} g(s) E_{\mu, \nu, l}^{\gamma, \delta, k}\left(\omega s^{\mu}\right) d s\right|^{\nu p} d t\right)^{\frac{1}{p}}\left(\int_{a}^{m b}\left|f^{\prime}(t)\right|^{q} d t\right)^{\frac{1}{q}} .
\end{aligned}
$$

By using $\|g\|_{\infty}=\sup _{t \in[a, b]}|g(t)|$ and absolute convergence of Mittag-Leffler function, we have

$$
\begin{aligned}
& \mid\left(\int_{a}^{m b} g(s) E_{\mu, \nu, l}^{\gamma, \delta, k}\left(\omega s^{\mu}\right) d s\right)^{\nu}(f(a)+f(m b)) \\
& -\nu \int_{a}^{m b}\left(\int_{a}^{t} g(s) E_{\mu, \nu, l}^{\gamma, \delta, k}\left(\omega s^{\mu}\right) d s\right)^{\nu-1} g(t) E_{\mu, \nu, l}^{\gamma, \delta, k}\left(\omega t^{\mu}\right) f(t) d t \\
& -\nu \int_{a}^{m b}\left(\int_{t}^{m b} g(s) E_{\mu, \nu, l}^{\gamma, \delta, k}\left(\omega s^{\mu}\right) d s\right)^{\nu-1} g(t) E_{\mu, \nu, l}^{\gamma, \delta, k}\left(\omega t^{\mu}\right) f(t) d t \mid \\
& \leq\|g\|_{\infty}^{\nu} S^{\nu}\left(\left(\int_{a}^{m b}|t-a|^{\nu p} d t\right)^{\frac{1}{p}} \mid\right. \\
& \left.+\left(\int_{a}^{m b}|m b-t|^{\nu p} d t\right)^{\frac{1}{p}}\right)\left(\int_{a}^{m b}\left|f^{\prime}(t)\right|^{q} d t\right)^{\frac{1}{q}} .
\end{aligned}
$$

Since $\left|f^{\prime}(t)\right|^{q}$ is $m$-convex, we have

$$
\left|f^{\prime}(t)\right|^{q} \leq \frac{m b-t}{m b-a}\left|f^{\prime}(a)\right|^{q}+\frac{m(t-a)}{m b-a}\left|f^{\prime}(b)\right|^{q} .
$$

Using (3.12) in (3.11), we have

$$
\begin{aligned}
& \mid\left(\int_{a}^{m b} g(s) E_{\mu, \nu, l}^{\gamma, \delta, k}\left(\omega s^{\mu}\right) d s\right)^{\nu}(f(a)+f(m b)) \\
& -\nu \int_{a}^{m b}\left(\int_{a}^{t} g(s) E_{\mu, \nu, l}^{\gamma, \delta, k}\left(\omega s^{\mu}\right) d s\right)^{\nu-1} g(t) E_{\mu, \nu, l}^{\gamma, \delta, k}\left(\omega t^{\mu}\right) f(t) d t \\
& -\nu \int_{a}^{m b}\left(\int_{t}^{m b} g(s) E_{\mu, \nu, l}^{\gamma, \delta, k}\left(\omega s^{\mu}\right) d s\right)^{\nu-1} g(t) E_{\mu, \nu, l}^{\gamma, \delta, k}\left(\omega t^{\mu}\right) f(t) d t \mid \\
& \leq\|g\|_{\infty}^{\nu} S^{\nu}\left(\left(\int_{a}^{m b}|t-a|^{\nu p} d t\right)^{\frac{1}{p}}+\left(\int_{a}^{m b}|m b-t|^{\nu p} d t\right)^{\frac{1}{p}}\right) \\
& \times\left(\int_{a}^{m b} \frac{m b-t}{m b-a}\left|f^{\prime}(a)\right|^{q}+\frac{m(t-a)}{m b-a}\left|f^{\prime}(b)\right|^{q}\right)^{\frac{1}{q}} .
\end{aligned}
$$


After a simple calculation, we get the required result.

Remark 3.7. It is remarkable that by taking particular values of parameters of MittagLeffler function in above theorem several fractional integral inequalities can be obtained for corresponding fractional integrals. For example some results are given below.

Corollary 3.8. In Theorem 3.6 if we take $m=1$, then we have the following integral inequality

$$
\begin{aligned}
& \mid\left(\int_{a}^{b} g(s) E_{\mu, \nu, l}^{\gamma, \delta, k}\left(\omega s^{\mu}\right) d s\right)^{\nu}(f(a)+f(b)) \\
& -\nu \int_{a}^{b}\left(\int_{a}^{t} g(s) E_{\mu, \nu, l}^{\gamma, \delta, k}\left(\omega s^{\mu}\right) d s\right)^{\nu-1} g(t) E_{\mu, \nu, l}^{\gamma, \delta, k}\left(\omega t^{\mu}\right) f(t) d t \\
& -\nu \int_{a}^{b}\left(\int_{t}^{b} g(s) E_{\mu, \nu, l}^{\gamma, \delta, k}\left(\omega s^{\mu}\right) d s\right)^{\nu-1} g(t) E_{\mu, \nu, l}^{\gamma, \delta, k}\left(\omega t^{\mu}\right) f(t) d t \mid \\
& \leq \frac{2(b-a)^{\nu+1}\|g\|_{\infty}^{\nu} S^{\nu}}{(\nu p+1)^{\frac{1}{q}}}\left(\frac{\left|f^{\prime}(a)\right|^{q}+\left|f^{\prime}(b)\right|^{q}}{2}\right)^{\frac{1}{q}} .
\end{aligned}
$$

Remark 3.9. In Theorem 3.6, for $m=1$.

(i) If we put $\omega=0$, then we get [19, Theorem 7].

(ii) If we take $\omega=0$ along with $\nu=\frac{\mu}{k}$, then we get [9, Theorem 2.5].

(iii) If we take $g(s)=1$ and $\omega=0$, then we get [3, Theorem 2.3].

(iv) If we put $\omega=0$ and $\nu=1$, then we get [3, Corollary 3$]$.

In the next result we give the Hadamard type inequalities for $m$-convex functions via generalized fractional integral operator containing generalized Mittag-Leffler function.

Theorem 3.10. Let $f:[a, m b] \rightarrow \mathbb{R}$ be a positive function with $0 \leq a<b$ and $f \in L[a, m b]$. If $f$ is $m$-convex function, then the following inequalities for generalized fractional integral hold

$$
\begin{aligned}
& f\left(\frac{a+m b}{2}\right)\left(\epsilon_{\mu, \nu, l, \omega^{\prime},\left(\frac{a+m b}{2}\right)+}^{\gamma, \delta, k}\right)(m b) \\
& \leq\left(\epsilon_{\mu, \nu, l, \omega^{\prime},\left(\frac{a+m b}{2}\right)+}^{\gamma, \delta, k}\right)(m b)+\left(\epsilon_{\mu, \nu, l, m^{\mu} \omega^{\prime},\left(\frac{a+m b}{2 m}\right)-}^{\gamma, \delta, k}\right)\left(\frac{a}{m}\right) \\
& \leq \frac{1}{m b-a}\left[f(a)-m f\left(\frac{a}{m^{2}}\right)\right]\left(\epsilon_{\mu, \nu+1, l, \omega^{\prime},\left(\frac{a+m b}{2}\right)+}^{\gamma, \delta, k}\right)(m b) \\
& +m^{\nu+1}\left(f(b)+m f\left(\frac{a}{m^{2}}\right)\right)\left(\epsilon_{\mu, \nu, l, m^{\mu} \omega^{\prime},\left(\frac{a+m b}{2 m}\right)+}^{\gamma, \delta, k}\right)\left(\frac{a}{m}\right)
\end{aligned}
$$

where $\omega^{\prime}=\frac{2^{\mu} \omega}{(m b-a)^{\mu}}$.

Proof. Using $m$-convexity of $f$, we have

$$
f\left(\frac{x+m y}{2}\right) \leq \frac{f(x)+m f(y)}{2}
$$


for $x, y \in[a, m b]$.

By taking $x=\frac{t}{2} a+\frac{2-t}{2} m b, y=\frac{2-t}{2 m} a+\frac{t}{2} b$ for $t \in[0,1]$ such that $x, y \in[a, m b]$, inequality (3.14) becomes

$$
2 f\left(\frac{a+m b}{2}\right) \leq f\left(\frac{t}{2} a+\frac{2-t}{2} m b\right)+m f\left(\frac{2-t}{2 m} a+\frac{t}{2} b\right)
$$

Multiplying both sides of (3.15) by $t^{\nu-1} E_{\mu, \nu, l}^{\gamma, \delta, k}\left(\omega t^{\mu}\right)$ and integrating with respect to $t$ on $[0,1]$

$$
\begin{aligned}
& 2 f\left(\frac{a+m b}{2}\right) \int_{0}^{1}\left(t^{\nu-1}\right) E_{\mu, \nu, l}^{\gamma, \delta, k}\left(\omega t^{\mu}\right) d t \\
& \leq \int_{0}^{1}\left(t^{\nu-1}\right) E_{\mu, \nu, l}^{\gamma, \delta, k}\left(\omega t^{\mu}\right) f\left(\frac{t}{2} a+\frac{2-t}{2} m b\right) d t \\
& +m \int_{0}^{1}\left(t^{\nu-1}\right) E_{\mu, \nu, l}^{\gamma, \delta, k}\left(\omega t^{\mu}\right) f\left(\frac{2-t}{2 m} a+\frac{t}{2} b\right) d t .
\end{aligned}
$$

Setting $u=\frac{t}{2} a+\frac{2-t}{2} m b$ and $v=\frac{2-t}{2 m} a+\frac{t}{2} b$ in (3.16), we have

$$
\begin{aligned}
& 2 f\left(\frac{a+m b}{2}\right) \int_{\frac{a+m b}{2}}^{m b}(m b-u)^{\nu-1} E_{\mu, \nu, l}^{\gamma, \delta, k}\left(\omega^{\prime}(m b-u)^{\mu}\right) d u \\
& \leq \int_{\frac{a+m b}{2}}^{m b}(m b-u)^{\nu-1} E_{\mu, \nu, l}^{\gamma, \delta, k}\left(\omega^{\prime}(m b-u)^{\mu}\right) f(u) d u \\
& +m^{\nu+1} \int_{\frac{a}{m}}^{\frac{a+m b}{2 m}}\left(v-\frac{a}{m}\right)^{\nu-1} E_{\mu, \nu, l}^{\gamma, \delta, k}\left(m^{\mu} \omega^{\prime}\left(v-\frac{a}{m}\right)^{\mu}\right) f(v) d v
\end{aligned}
$$

where $\omega^{\prime}=\frac{2^{\mu} \omega}{(m b-a)^{\mu}}$.

This implies

$$
\begin{aligned}
& 2 f\left(\frac{a+m b}{2}\right)\left(\epsilon_{\mu, \nu, l, \omega^{\prime},\left(\frac{a+m b}{2}\right)+}^{\gamma, \delta, k} 1\right)(m b) \\
& \leq\left(\epsilon_{\mu, \nu, l, \omega^{\prime},\left(\frac{a+m b}{2}\right)^{\gamma}}^{\gamma, \delta, k}\right)(m b)+\left(\epsilon_{\mu, \nu, l, m^{\mu} \omega^{\prime},\left(\frac{a+m b}{2 m}\right)_{-}}^{\gamma, \delta, k} f\right)\left(\frac{a}{m}\right) .
\end{aligned}
$$

To prove the second inequality from $m$-convexity of $f$, we have

$$
\begin{aligned}
& f\left(\frac{t}{2} a+m \frac{2-t}{2} b\right)+m f\left(\frac{2-t}{2 m} a+\frac{t}{2} b\right) \\
& \leq \frac{t}{2}\left(f(a)-m^{2} f\left(\frac{a}{m^{2}}\right)\right)+m\left(f(b)+m f\left(\frac{a}{m^{2}}\right)\right) .
\end{aligned}
$$


Multiplying both sides of (3.19) by $t^{\nu-1} E_{\alpha, \beta, l}^{\gamma, \delta, k}\left(\omega t^{\alpha}\right)$ and integrating with respect to $t$ over $[0,1]$, we have

$$
\begin{aligned}
& \int_{0}^{1} t^{\nu-1} E_{\mu, \nu, l}^{\gamma, \delta, k}\left(\omega t^{\mu}\right) f\left(\frac{t}{2} a+m \frac{2-t}{2} b\right) d t \\
& +m \int_{0}^{1} t^{\nu-1} E_{\mu, \nu, l}^{\gamma, \delta, k}\left(\omega t^{\mu}\right) f\left(\frac{2-t}{2 m} a+\frac{t}{2} b\right) \\
& \leq \frac{1}{2}\left(f(a)-m^{2} f\left(\frac{a}{m^{2}}\right)\right) \int_{0}^{1} t^{\nu} E_{\mu, \nu, l}^{\gamma, \delta, k}\left(\omega t^{\mu}\right) d t \\
& +m\left(f(b)+m f\left(\frac{a}{m^{2}}\right)\right) \int_{0}^{1} t^{\nu-1} E_{\mu, \nu, l}^{\gamma, \delta, k}\left(\omega t^{\mu}\right) d t .
\end{aligned}
$$

Setting $u=\frac{t}{2} a+m \frac{2-t}{2} b$ and $v=\frac{2-t}{2 m} a+\frac{t}{2} b$ in (3.20), we have

$$
\begin{aligned}
& \int_{\frac{a+m b}{2}}^{m b}(m b-u)^{\nu-1} E_{\mu, \nu, l}^{\gamma, \delta, k}\left(\omega^{\prime}(m b-u)^{\mu}\right) f(u) d u \\
& +\int_{\frac{a}{m}}^{\frac{a+m b}{2 m}}\left(v-\frac{a}{m}\right)^{\nu-1} E_{\mu, \nu, l}^{\gamma, \delta, k}\left(m^{\mu} \omega^{\prime}\left(v-\frac{a}{m}\right)^{\mu}\right) f(v) d v \\
& \leq \frac{1}{2}\left(f(a)-m^{2} f\left(\frac{a}{m^{2}}\right)\right) \int_{\frac{a+m b}{2}}^{m b}(m b-u)^{\nu} E_{\mu, \nu, l}^{\gamma, \delta, k}\left(\omega^{\prime}(m b-u)^{\mu}\right) d t \\
& +m^{\nu+1}\left(f(b)+m f\left(\frac{a}{m^{2}}\right)\right) \int_{\frac{a}{m}}^{\frac{a+m b}{2 m}}\left(v-\frac{a}{m}\right)^{\nu-1} E_{\mu, \nu, l}^{\gamma, \delta, k}\left(m^{\mu} \omega^{\prime}\left(v-\frac{a}{m}\right)^{\mu}\right) d t .
\end{aligned}
$$

This implies

$$
\begin{aligned}
& \left(\epsilon_{\mu, \nu, l, \omega^{\prime},\left(\frac{a+m b}{2}\right)^{\gamma, \delta, k}}^{+} f\right)(m b)+m^{\nu+1}\left(\epsilon_{\mu, \nu, l, m^{\mu} \omega^{\prime},\left(\frac{a+m b}{2 m}\right)_{-}^{\gamma, \delta, k}}^{f}\right)\left(\frac{a}{m}\right) \\
& \leq \frac{1}{m b-a}\left(f(a)-m^{2} f\left(\frac{a}{m^{2}}\right)\right)\left(\epsilon_{\mu, \nu+1, l, \omega^{\prime},\left(\frac{a+m b}{2}\right)^{\gamma, \delta, k}}^{1}\right)(m b) \\
& +m^{\nu+1}\left(f(b)+m f\left(\frac{a}{m^{2}}\right)\right)\left(\epsilon_{\mu, \nu, l, m^{\mu} \omega^{\prime},\left(\frac{a+m b}{2 m}\right)_{-}^{\gamma, \delta}}^{\gamma} 1\right)\left(\frac{a}{m}\right) .
\end{aligned}
$$

Combining (3.18) and (3.22) we get the result.

Corollary 3.11. In Theorem 3.10 if we take $\omega=0$, then we get the following inequality for Riemann-Liouville fractional integral operator

$$
\begin{aligned}
& f\left(\frac{a+m b}{2}\right) \leq \frac{2^{\nu-1} \Gamma(\nu+1)}{(m b-a)^{\mu}}\left(I_{\left(\frac{a+m b}{2}\right)^{+}}^{\nu} f(m b)+m^{\nu+1} I_{\left(\frac{a+m b}{2 m}\right)_{-}}^{\nu} f\left(\frac{a}{m}\right)\right) \\
& \leq \frac{\nu}{4(\nu+1)}\left(f(a)-m^{2} f\left(\frac{a}{m^{2}}\right)\right)+\frac{m}{2}\left(f(b)+m f\left(\frac{a}{m^{2}}\right)\right) .
\end{aligned}
$$

Remark 3.12. If we put $\omega=0, m=1$ and $\nu=1$ in Theorem 3.10, then we get the classical Hadamard inequality.

Acknowledgement. The research work of Ghulam Farid is supported by the Higher Education Commission of Pakistan under NRPU 2016, Project No. 5421. 


\section{References}

[1] Dalir, M., Bashour, M., Applications of fractional calculus, Appl. Math. Sci., 4(2010), no. 21, 1021-1032.

[2] Dragomir, S.S., On some new inequalities of Hermite-Hadamard type for $m$-convex functions, Turkish J. Math., 33(2002), no. 1, 45-55.

[3] Dragomir, S.S., Agarwal, R.P., Two inequalities for differentiable mappings and applications to special means of real numbers and trapezoidal formula, Appl. Math. Lett., 11(1998), no. 5, 91-95.

[4] Dragomir, S.S., Toader, G.H., some inequalities for $m$-convex functions, Stud. Univ. Babeş-Bolyia. Math., 38(1993), no. 1, 21-28.

[5] Farid, G., A treatment of the Hadamard inequality due to m-convexity via generalized fractional integrals, J. Fract. Calc. Appl. , 9(2018), no. 1, 8-14.

[6] Farid, G., Hadamard and Fejér-Hadamard inequalities for generalized fractional integrals involving special functions, Konuralp J. Math., 4(2016), no. 1, 108-113.

[7] Farid, G., Marwan, M., Rehman, A.U., New mean value theorems and generalization of Hadamard inequality via coordinated $m$-convex functions, J. Inequal. Appl., Article ID 283, (2015), 11 pp.

[8] Farid, G., Pečarić, J., Tomovski, Z., Opial-type inequalities for fractional integral operator involving Mittag-Leffler function, Fract. Differ. Calc., 5(2015), no. 1, 93-106.

[9] Farid, G., Rehman, A.U., Generalizations of some integral inequalities for fractional integrals, Ann. Math. Sil., to appear, https://doi.org/10.1515/amsil-2017-0010.

[10] Iscan, I., New estimates on generalization of some integral inequalities for $(\alpha, m)$-convex functions, Contemp. Anal. Appl. Math., 1(2013), no. 2, 253-264.

[11] Kilbas, A.A., Srivastava, H.M., Trujillo, J.J., Theory and applications of fractional differential equations, North-Holland Mathematics Studies, 204, Elsevier, New York-London, 2006.

[12] Laurent, H., Sur le calcul des derivees a indices quelconques, Nouv. Annales de Mathematiques, 3(1884), no. 3, 240-252.

[13] Lazarević, M., Advanced topics on applications of fractional calculus on control problems, System Stability and Modeling, WSEAS Press, 2014.

[14] Letnikov, A.V., Theory of differentiation with an arbitray index (Russian), Moscow, Matem. Sbornik, 3, 1-66, 1868.

[15] Miller, K., Ross, B., An introduction to the fractional differential equations, John, Wiley and Sons Inc., New York, 1993.

[16] Mocanu, P.T., Şerb, I., Toader, G., Real star convex functions, Stud. Univ. Babeş-Bolyai Math., 42(1997), no. 3, 65-80.

[17] Prabhakar, T.R., A singular integral equation with a generalized Mittag-Leffler function in the kernel, Yokohama Math. J., 19(1971), 7-15.

[18] Salim, L.T.O., Faraj, A.W., A Generalization of Mittag-Leffler function and integral operator associated with integral calculus, J. Frac. Calc. Appl., 3(2012), no. 5, 1-13.

[19] Sarikaya, M.Z., Erden, S., On the Hermite-Hadamard Fejér type integral inequality for convex functions, Turkish Journal of Analysis and Number Theory, 2(2014), no. 3, 85-89.

[20] Sarikaya, M.Z., Set, E., Yaldiz, H., Basak, N., Hermite-Hadamard inequalities for fractional integrals and related fractional inequalities, J. Math. Comput. Model, 57(2013), 2403-2407. 
[21] Sonin, N.Y., On differentiation with arbitray index, Moscow Matem. Sbornik, 6(1869), no. 1, 1-38.

[22] Srivastava, H.M., Tomovski, Z., Fractional calculus with an integral operator containing generalized Mittag-Leffler function in the kernal, Appl. Math. Comput., 211(2009), no. 1, 198-210.

[23] Toader, G., Some generaliztion of convexity, Proc. Colloq. Approx. Optim, Cluj Napoca (Romania), (1984), 329-338.

[24] Tomovski, Z., Hiller, R., Srivastava, H.M., Fractional and operational calculus with generalized fractional derivative operators and Mittag-Leffler function, Integral Transforms Spec. Funct., 21(2011), no. 11, 797-814.

Ghulam Farid

COMSATS Institute of Information Technology, Attock Campus

Department of Mathematics

Attock, Pakistan

e-mail: ghlmfarid@ciit-attock.edu.pk, faridphdsms@hotmail.com

Ghulam Abbas

University of Sargodha, Department of Mathematics

Sargodha, Pakistan

and

Government College Bhalwal, Department of Mathematics

Sargodha, Pakistan

e-mail: prof .abbas6581@gmail.com 